\title{
Tres tratados médicos de la Ets Haim: entre traducciones, apuntes enciclopédicos y polémicas médicas del siglo XVII
}

\author{
The Est Haim medical treatises: between \\ translations, encyclopedic notes \\ and medical controversies on XVII century
}

\author{
Fernando J. Pancorbo \\ Universität Basel, Suiza
}

ORCID: 0000-0002-2557-4428

fernandojose.pancorbo@unibas.ch

\section{RESUMEN}

El presente trabajo tiene como objetivo ofrecer una revisión de la historiografía médica que se desarrolló en el contexto de la comunidad sefardí de Ámsterdam a lo largo del siglo XVII. A través de tres manuscritos, que se encuentran albergados en la biblioteca judía Ets Haim, la biblioteca judía más antigua del mundo, muestro el interés que suscitan estas obras -prácticamente desconocidas-, ya no sólo por la cuestión propiamente clínica, sino porque además cubren una laguna importante en cuanto a la herencia y transmisión de saberes, además de la constatación de la existencia de redes culturales en las que los sefardíes occidentales jugaron un papel primordial. Fruto de esta interrelación de doctos y de relaciones basadas en el interés cultural, surgieron, paralelamente, algunos escritos polémicos, centrados puramente en la materia científica, como el que presento junto a esos tres manuscritos. Se trata de un opúsculo -escrito en latín y anexado a otras páginas de una polémica completamente ajena 
a la materia médica- cuyas pistas abren la hipótesis de su muy probable atribución al físico sefardí Zacuto Lusitano. Es, pues, el interés de este estudio ofrecer nuevas líneas de investigación y de trabajo.

Palabras clave: historiografía médica; manuscritos; Ets Haim; sefardíes; Ámsterdam; Zacuto Lusitano.

\section{ABSTRACT}

The aim of this paper is to offer a review of the medical historiography that developed in the context of the Sephardic community of Amsterdam during the seventeenth century. By means of three manuscripts, housed in the Jewish library Ets Haim, the oldest Jewish library in the world, I show the interest that these practically unknown works arouse, not only because of the clinical issue itself, but also because they fill an important gap in terms of the inheritance and transmission of knowledge, as well as the confirmation of the existence of cultural networks in which the Western Sephardim played a key role. As a result of this interrelation of scholars and relations based on cultural interest, some polemical writings arose at the same time, focused purely on scientific matters, such as the one I present next to these three manuscripts. It is an opuscule -written in Latin and appended to other pages of a polemic completely unrelated to medical matters- whose clues open the hypothesis of its very probable attribution to the Sephardic physicist Zacuto Lusitano. It is therefore in the interest of this study to offer new lines of research and work.

Keywords: medical historiography; manuscripts; Ets Haim; Sephardim; Amsterdam; Zacuto Lusitanus.

En esta ocasión, mi atención se centra en el estudio de los manuscritos de contenido medicinal que se conservan digitalizados en la biblioteca judía de Ets Haim, ${ }^{1}$ de Ámsterdam, ya que son de

${ }^{1}$ La Ets Haim es la biblioteca judía más antigua del mundo aún en funcionamiento, otrora la primera yeshivá instituida en la comunidad sefardí de Ámsterdam -más concretamente, en la primera mitad del siglo XvII- y, sin duda, una de las más importantes de la diáspora de Europa occidental. En su interior, además de volúmenes impresos de diferente naturaleza, alberga manuscritos centrados en intereses de diverso interés, más allá de los propiamente ligados a la fe mosaica -como libros de la Kabbalah, la Halakhah, 
gran interés, aunque no han sido estudiados en profundidad prácticamente en ninguno de los casos. Tanto es así que sólo han sido mencionados como curiosidad, o como dato anecdótico, en algunos volúmenes de Historia de la medicina o en revisiones de carácter bibliográfico o literario, a pesar de que algunos de ellos se hayan llegado a reconocer en los últimos años como obras pioneras de su época (Fuks \& Fuks-Mansfeld, 1975; Arrizabalaga, 2009). Su escasa trascendencia parece haberse visto mermada por diferentes razones (Baudry, 2020): en primer lugar, porque ninguna de ellas fue impresa, aspecto que, lógicamente, dificulta su difusión; en segundo lugar, porque el hecho de que estén escritos en lengua portuguesa o traducidos a ella -salvo las polémicas, que estaban redactadas en su mayoría en latín- ${ }^{2}$ evidencian que, originariamente, estaban pensados y destinados a un público, en cierto modo, familiar o cercano; y en tercer lugar, porque se entienden como escritos de carácter enciclopédico o cuadernos de notas. No obstante, y superando estas barreras, o prejuicios, tales documentos han de entenderse desde una perspectiva mucho más amplia, teniendo en cuenta que, por un lado, muestran una relación directa con la tradición médica local, así como con la internacional -rompiendo estas fronteras invisibles que han aislado a estos textos-, y que, gracias a los movimientos migratorios y las interacciones de estos doctos fuera de los muros de su comunidad, comportan un obvio e importantísimo intercambio de conocimientos y de tradiciones médicas, y, por otro, que en algunos casos las obras de estos médicos y cirujanos fueron, sin duda, primera piedra o vehículo de nuevos saberes allá donde fueran, incluso en ultramar, como se verá.

Centrándome en el caso concreto de la Ets Haim, cabe decir que sólo se encuentran digitalizados tres manuscritos, cuyo con-

\footnotetext{
el Midrash, etc.-, como astronomía y cronología, literatura, polémicas surgidas en torno a retórica, filosofía, o teología. A pesar de que su historia ha sido ampliamente estudiada, ofrezco aquí algunos estudios que considero lecturas de referencia: Barrios (1683, p. 90), Berger (1996), Bergmann (2006).

${ }^{2}$ Que se redactasen en latín puede deberse a que fueron textos concebidos en entornos académicos, al contrario que buena parte de los manuscritos que se conservan, muy probablemente destinados a un uso privado o al provecho entre correligionarios.
} 
tenido es propiamente médico: un Catálogo de remedios, redactado por David Aboab en 1685; el Tratado sobre medecina que fez o Doutor Zacuto, hacia 1690; y, por último, un Libelo aureo da difficultade de ourinar, redactado por Semuel Leão de Benavente en 1699. ${ }^{3}$ Sin embargo, y a pesar de que abordaré estos tres ejemplares, el interés de este trabajo se centra, además, en el comentario y atribución de una polémica desconocida, fuera de catálogo, y que se encuentra localizada como anexo a una colección de escritos polémicos, que responden a una misma apología, pero que no guardan ninguna relación con la medicina.

Con respecto a los tres ejemplares recogidos y catalogados, diré que reúnen una serie de detalles que permiten observarlos a modo de brevísimo corpus, ya que todos ellos están escritos en portugués y tienen como punto en común la ligazón paradójica con la figura de Ishak de Matatia Aboab (Kayserling, 1890, pp. 3-4; Löwestein, 1904, pp. 661-701; Révah, 1961, pp. 276-310; McGaha, 1998, pp. 226-317; Pancorbo, 2021, pp. 33-49). De Matatia fue un eminente poeta, dramaturgo y figura destacada entre los sefardíes amstelodamos del siglo XVII, pero cuya única relación con la medicina fue su hijo, David Aboab, que fue quien realmente ejerció esta ciencia (Roth, 2007, II, p. 265; Oliel-Grausz, 2017a, pp. 89-118). De este último, existe un escueto Catálogo de diferentes remedios para diversas sortes de achaques (Fuks \& Fuks-Mansfeld, 1975, II, p. 183), cuya apariencia es la de un compendio de notas, en el que recoge diferentes curas, acompañadas de una descripción empírica, que, a lo largo de sus capítulos, evidencia con el subtítulo de "es remedio de experiencia aprobada". La curiosidad de este texto reside en que las soluciones que plantea, y que según él dan buen resultado, son una colección de métodos que ha recogido de la tradición médica propia de su entorno cultural y religioso -como el modo de parar las hemorragias por la circuncisión-, así como del local. En este aspecto, esta últimas -es decir, las ajenas a su condición- son las

${ }^{3}$ No voy a ofrecer aquí la descripción física de los manuscritos, ya que se pueden consultar, bien en la propia página de la Ets Haim -<https://etshaimmanuscripts.nl/ collection/manuscripts/>- o bien en Fuks \& Fuks-Mansfeld (1975, II, pp. 182-183). 
que brindan mayor interés, ya que, si por un lado colige remedios aprendidos de otros doctores, como el purgativo, proporcionado por el «doutor Reinoso», ${ }^{4}$ o como los ingredientes que receta directamente en lengua holandesa-Brande win, poek hout, penwortel, etc.-, hay otros remedios que chocan de manera directa con su fe y que, a fuerza, debieron ser tomados de tratados o testimonios ajenos al entorno de la comunidad judía, como la administración de cuatro granos de armónico para purgar y deshacer los embarazos de poco tiempo. ${ }^{5}$ Desgraciadamente, no existe constancia ni mención alguna a este texto, por lo que lo más razonable es pensar que, efectivamente, fuese un cuaderno de apuntes personal.

Caso semejante es el de Semuel Leão Benavente, ${ }^{6}$ el primer judío que fue admitido de manera oficial en el registro de gremios como cirujano, ${ }^{7}$ y su primer libelo, centrado en las anomalías urinarias y en la litotomía. Este ejemplar es un breve tratado, en el que el célebre físico judío -además de notable hombre de letras- expone curas y praxis recogidas, fundamentalmente, de las lecciones que recibió del médico parisino François Collot $^{8}$-maestro también de

4 "Reseita de hum lambedor purgativo, couza rara, e bom de tomar, ordenado pello D. Reinoso. E tomando duas culheras deste lambedor por vez, he bastante para purgar" (f. 24 v).

${ }^{5}$ Explicita lo siguiente en su Catálogo de remedios: "4 grãos de antimonio preparado faz purgamento, e desfazer o prenhado de pouco tempo" (f. 25 r).

${ }^{6}$ Apenas hay algunas referencias de época sobre la figura de este médico y cirujano, como las que ofrece Miguel de Barrios (1683) en sus Triumphos del gobierno popular, donde se refiere a él como "Doutor Semuel Benavente, chirurgão famoso et boticario perito en varias sciencias, et linguas, et celebre poeta". Posteriormente, recuperará algunos de sus datos David Franco Mendes (1976), en sus Memorias do estabelecemento e progresso dos judeos (pp. 80-84), entre los que se puede ver que, además, fue uno de los médicos más destacados de la comunidad y que prestó sus servicios a la Tsedaka. El estudio más detallado sobre la vida y las aportaciones médicas de Semuel Leão Benavente es el de Cohen (1930, pp. 2234-2256) y el de I. van Esso Bzn, "Het aandeel der Joden in the natuurwetenschappen in der Nederland" (1940, pp. 665-666). En cuanto a las referencias a Giovanni de Vigo y Avicena, reseño las que él mismo realiza a lo largo de varios pasajes de su obra.

${ }^{7}$ El segundo sería su yerno: David Baruch Benavente. Fueron los únicos que estuvieron colegiados oficialmente, aunque de manera tardía, ya que no lo se hizo oficial hasta 1710 , tal y como se puede ver en Da Silva Rosa (1925, p. 105).

${ }^{8}$ François Collot fue un médico y cirujano, que continuó una larga tradición familiar, dedicada a las litotomías. Los descubrimientos e innovaciones que hizo el francés sobre 
otros sefardíes, como David Cardoso o Josseph Leví Flores $-{ }^{9}$ y de la tradición médica de autores anteriores, como Giovanni de Vigo o, remontándose aún más en el tiempo, Avicena (Arrizabalaga, 2007, pp. 1-24). Es el único ejemplar que está ilustrado, probablemente a partir de los diseños que haría en ese tiempo François o alguno de sus familiares o colegas de profesión, aspecto que demuestra que, si en un principio pudiese ser concebido para un entorno cercano, el objetivo final parece estar destinado a su impresión, aunque nunca llegó a ser así. Más allá, este Libello aureo da difficultade de ourinar es el segundo texto que está relacionado con la familia Aboab -pues la dedicatoria así lo hace ver, así como en la cubierta del libro, donde aparece el nombre de David Aboab Curiel-, cuestión que no es extraña si se tiene en cuenta que Isaac de Matatiah fue reconocido en diversas ocasiones como recopilador "de diferentes materias, principalmente de interés judaico, pero también de saberes más generales" (Den Boer, 1995; Pancorbo, 2021).

$\mathrm{El}$ tercer manuscrito, el Tratado sobre medecina que fez o doutor $\mathrm{Za}$ cuto, ${ }^{10}$ responde también a las inquietudes y curiosidades bibliófilas de Isaac de Matatiah, ya que éste encargó a Benjamín Godínez

los métodos de extracción de las piedras renales fueron ampliamente difundidas -beneficiando, entre otros, a Semuel Leão-, aunque sus enseñanzas no serían publicadas, póstumamente, hasta 1727, bajo el título de Traité de l'opération de la taille. Véase Cohen (1930, pp. 2241-2242). En el manuscrito del Libello aureo da difficultade de ourinar, se puede leer lo siguiente: "Com que darei fim á discripção dos causticos com aquelle que me ensinou François Collo [sic], o qual, nesse tempo, mo deo per invenção propria, porem eo achei em autores alguns que se parecem a elle, mas como me achasse sempre com elle, ão quis pôr em perigo algum patiente por experimentar novos medicamentos onde o certo he melor que o duvidozo, e mais sendo fazil de fazer, como segue" (ff. 75-76).

${ }^{9}$ Sobre la praxis de estos dos médicos sefardíes, Leão da algunas pistas, pero no muy elogiosas. De ellos, cuenta lo siguiente: "He de nottar que esta cura [de las carnosidades] toda consiste en non purgar bem de materia pella chaga e todo o secreto desta cura he o dilatar o collo da bexiga, porque havendo nesse lugar alguas carnosidades duras [...] com o dilatar se rompem, e se desfazem en materia, como vi em David Cardoso e Josseph Leví Flores, que ambas operaçõens asesti e curie despois de havellos aberto meo mestre Collo [sic]" (f. 101).

${ }^{10}$ Existe una edición moderna, realizada por Ana Carolina de Carvalho y Gabriel Ferreira Gurian, en 2018. 
-otro hombre de letras, aunque poco conocido- ${ }^{11}$ que copiara la obra del Zacuto Lusitano, en 1690, con el fin de que se lo llevase su hijo David, como manual de primeros auxilios, en su viaje a Brasil (Oliel-Grausz, 2017b, pp. 159-182). Es, en sí, curiosidad que, de forma paralela, pone de relieve aquello de lo que se quejó Cecil Roth cuando dijo que "no se ha tenido en cuenta la de ningún modo despreciable participación en el descubrimiento de América, que puede atribuirse a judíos profesos, tales como los dos científicos Abraham Zacuto, el astrónomo -antecesor del que aquí me ocupa-, y Crescas, el 'judío-mapa', y a algunos financieros como Abraham Senior" (Cecil Roth, 1979, p. 186).

Antes de hacer una breve descripción en este manuscrito y meterme de lleno en el tratamiento de la polémica, creo necesario hacer una breve reseña biográfica de este médico, ya que, además de que ha merecido el interés de recientes estudios, va a servir para contextualizar y dar explicación a estos dos escritos. En sí, dos han sido los autores que se han interesado por la vida y la obra de Abraham Zacuto: el primero, Ludovico (Luiz) de Lemos (1907), cuyas notas biográficas, relativamente escuetas, se encuentran incluidas en los prolegómenos a la edición de su Opera Omnia del médico, más concretamente en la edición lionesa de 1642; y el segundo, Maximiano Lemos, eminente médico portugués e historiador de la medicina de la universidad de Oporto, que fue quien más se preocupó por estudiar la vida del judío y que materializó, en 1909, en su obra Zacuto Lustiano. A sua vida e a sua obra. Parto de estos dos trabajos para dar algunos retazos biográficos. ${ }^{12}$

Abraham Zacuto nació en 1575, en Lisboa, y estudió filosofía y medicina en las universidades de Coímbra y de Salamanca. A la

${ }^{11}$ Según se puede leer en el estudio de Constance Harris (2008): "Benjamin Godines, a calligrapher, editor, and printmaker, engraved a frontispiece for the me'ah Bracchot (Hundrer Blessing) in 1687”' (p. 101). Entre sus trabajos como calígrafo, se sabe que copió varias obras para Isaac de Matatia y para otros intelectos de la comunidad sefardí de Ámsterdam.

${ }^{12}$ Además, hay varios estudios que, aunque algo antiguos, siguen siendo referencias en lo relativo a la vida de este médico: Harry Friedenwald (1939, pp. 458-485), Szancer (1967, pp. 509-514), Jarcho (1989, pp. 291-295). 
edad de 21 años, consiguió el título de doctor por la Universidad de Sigüenza, institución que era una de las más reconocidas en la España de la época. Tras su estancia en tierras españolas, volvió a Lisboa, muy probablemente por la amenaza inquisitorial, donde durante cerca de treinta años estuvo ejerciendo su profesión. A pesar de que se ganó el respeto de sus conciudadanos, ya por su admirable praxis y dedicación al oficio, ya por su talante docto y de hombre de letras, el recrudecimiento de la actividad de la Inquisición -sobre todo a partir de 1547, año en el que el rey João III, apoyándose en la aprobación que hizo el papa Clemente VII, en 1531, estableció un tribunal a semejanza del español- le obligó a exiliarse en Ámsterdam. ${ }^{13}$ Llegó a la capital de las Provincias Unidas a la edad de 50 años, época en la que se circuncidó y en la que empieza a usar el apodo de "Lusitanus" - por cierto, algo muy común entre los doctos allí establecidos-; ${ }^{14}$ y de manera inminente, comenzó a destacar, ya no sólo entre los médicos y cirujanos de este entorno, sino también entre los hombres de letras y de fe (Brown, 2000, pp. 227-253). Es el caso de Menasseh ben Israel (1633), quien ofrece una carta de elogio publicada en El conciliador, que plasma la imagen de Abraham Zacuto como una eminencia, dedicado, además, al estudio de los saberes judíos (III-IV) -véase también Librach (1960, pp. 256-58) y Moreno de Carvalho (1996, pp. 147-159). En este sentido, se puede ver que el mismo doctor, en la peroratio al primer volumen de su Opera omnia, afirma lo siguiente:

Yo soy un judío y un extraño que huyó de Portugal y de mi más amado y encantador lugar de nacimiento, Lisboa, sacudido de un lado a otro por la grave desgracia y por las tormentas de una larga

\footnotetext{
${ }^{13}$ Franco Mendes (1976) recoge lo siguiente, en relación a su establecimiento en Ámsterdam: "Nesse anno 1614 largou o famoso Dr. Abraham Zacuto Luzitano (quem naceo no anno 1576) a honoriffica \& lucrativa professão que fazia de ser Insigne Medico, em Lixboa para observar a Ley Divina nesta cidade [Ámsterdam], donde exerceo a medecina que descreveo em 3 volumens, que admirarão as universidades da Europa sendo com author classico citado em todas ellas" (p. 22).

${ }^{14}$ Tal y como explica Maclean (2009), es frecuente encontrar entre los médicos de origen luso el apelativo de "peritus lusitanos" asociado a quienes pertenecían al gremio (pp. 371-401).
} 
vida... No he permitido que pase ningún día -como dice Sénecasin escribir una línea que mostrara mi amor por la República de la Medicina... Estaba decidido a dedicar mi vida a la devoción por el estudio (Zacuto, 1642). ${ }^{15}$

Y es que la visión que tenía Abraham Zacuto de su profesión era doble, ya que si, por un lado, su principal interés debía ser curar las enfermedades físicas, por otro, reafirmó, en varias de sus obras, la vinculación entre las anomalías de soma, psique y pathos, ya que tanto el estado psicológico y las consideraciones religiosas y sociales suponían evidentes condicionantes para el paciente y su evolución sanitaria.

Esta conexión entre saberes es realmente difícil de ver en el manuscrito que recoge la traducción, ya que responde a una selección de primeros auxilios y de curas escogidas, probablemente las más fáciles de sufrir durante las travesías marítimas, y la llegada a Brasil (Moreno de Carvalho, 1999, pp. 57-74). De hecho, esto se puede ver aún más claro si se atiende al subtítulo del primer capítulo: "Breve compendio en el cual se contiene la cura de todas las dolencias que acometiesen en el cuerpo humano, con el que te sabrás manejar durante el tiempo que no haya médico." 16 Sólo por medio de una carta de Isaac de Matatiah a su "Amado filho David Aboab Curiel" se pueden establecer algunos puentes con la perspectiva múltiple desde la que Zacuto aborda las enfermedades, concretamente en tres puntos. El primero, relativo a la deontología propia de la medicina, en la que hace una breve expositio, fundamentada en la Torah y en las obligaciones del juramento hipocrático -que,

15 "Nam Hebraeus sum, \& peregrinus qui deserens Lusitaniam, \& dulcissimam patriam Olysipponem, crebrioribus aerumnarum aetatisque longaeuae procellis, huc illucque, [...]. Tamen in medicam artem semper pronus perseveravi, inconcusso animo invictus, neque pertransit (ut ait Seneca) dies sine línea, in quo Republicam Medicam, quam perenni, et genuino amore ex toto corde redamo, non illustratim et sic diu, importune, vigilanti cura, sedulo labore, inexhausta diligentia, perfecto Medico \& arte Hippocratica digno decorum iudicavi studium abrumpere vitam" (Zacuto, 1642, p. 984). La traducción es mía.

16 "Compendio breve em o qual se contem a cura de todas as doenças que acometessem no corpo humano, com que te saberás governar em tempo que não haja medico." La traducción es mía. 
a pesar de que pueda parecer anacrónico, ya fue cumplido y jurado desde el Renacimiento en diversas academias médicas (Sousa Dias, 2010, pp. 77-88)-, que concuerdan además con las bases teóricas del tratado de Zacuto. El segundo, la cuestión relativa a la praxis y las interferencias con la fe, en la que Isaac de Matatiah aclara lo siguiente con respecto a cómo debe actuar su hijo:

Si bien soy consciente de que no es este el momento, a este propósito, haga diligencia el médico, aunque en Italia sea ley el hacerlo y en la cristiandad obligación advertirlo, obraréis en esto como a vos os parezca, y la ocasión y la urgencia lo pida, y el Señor Dios ponga bendición en la obra de vuestras manos, y enseñe a acertar como pude y como deseas (Zacuto, 1642, f. 100 v.). ${ }^{17}$

Es decir, que proceda según sus propios conocimientos y su conciencia profesional y personal, más allá de los posibles prejuicios religiosos existentes. $\mathrm{Y}$ en tercer lugar, el punto que más interés despierta y que sirvió de abono para diferentes suertes de polémicas: la diagnosis de enfermedades especulativas a partir de la categorización de las causas interiores y exteriores, ilustrado por un episodio que sufrió el propio Isaac de Matatiah, en el que pone en evidencia los errores que cometieron tres médicos con él, precisamente, para que su hijo no caiga en los mismos errores. ${ }^{18}$

Esta contemplación de todos los componentes, digamos, meta-medicinales sobre los que apoya sus estudios Abraham Zacuto fueron bastante discutidos tanto por sus contemporáneos como

17 "Se bem conheço que não será momento, a propósito, faça a diligencia o médico, ainda que em Itália é Escama o faça, e na cristandade obrigação adverti-lo, vos obrareis nisto como vos parecer, e a ocasião e urgência o pedir, e o Senhor Deus ponha Bênção na obra de vossas mãos, e ensine a aceitar como pede e desejas." La traducción es mía.

18 "Por quanto a my me sucedeo em huma grave doença, que achandome milhor della, huma noyte estando sô, e tendo huma 'romam' (que me havião trazido de regalo) comy parte della. A outro dia pella manha vierão os medicos que erão tres, e tomandome o pulso, e mandándome mostrar a lingua, e vendoma negra, sem preguntarme nada, comesarão a falar latin, noteficandome logo humas ventozas sarjadas, dizendome erão para asegurar alguma malenidade que pudese haver interior, e que fosse servido não fazer argumentos, como sempre solia, por quanto elles tratavão de mina saude, que muyto es" (ff. 92 v.- 93 r.). 
por tratadistas posteriores, en base a varios aspectos. El primero, quizás el más reseñable, es que todos los elementos teóricos que detalla en sus obras van adornados con cartas de colegas y con elementos retóricos, literarios y, en algunos casos, religiosos, que, a vista de sus contrarios, restan veracidad y objetividad a sus postulaciones. Esto se acentúa aún más si se tiene en cuenta que alguna de sus obras fue usada como fuente para la curación de posesiones demoníacas, como se puede ver en el tomo tercero de la obra de Joseph Bizouard (1863), Des rapports de l'homme avec le démon (III, pp. 505-506), en la que se puede ver que se cita un pasaje tomado del texto del médico judío: Praxis medica admiranda (1. III, obs. 139). En segundo lugar, y como consecuencia de la contaminación entre conocimientos, las discusiones relativas a los casos de enfermedades especulativas llegaron a tomar unas derivas de carácter personal, enfocadas al razonamiento relativo a las condiciones psíquicas y a los condicionantes externos de los enfermos, lo cual constituía un área de disensión bastante subjetiva. Son varios los ejemplos estudiados, y que dan buena fe de estas dicotomías sociales y religiosas alrededor de la materia médica, como la mentira por parte del enfermo, la preeminencia del remedio físico por encima del mental o la mera consecuencia de ser judíos, tal y como se puede ver, por ejemplo, a lo largo del detallado estudio que Winfried Schleiner (1995) hace sobre esta cuestión (pp. 78-94).

No obstante, y a pesar de que la vida y la obra de Abraham Zacuto ha despertado un gran interés en los últimos años, siguen apareciendo documentos relacionados con sus textos y con las discordancias surgidas entre los especialistas de la materia. Es el caso de Arrogantia convicta, un brevísimo texto anexado a una polémica de carácter retórico - desarrollada con seguridad a comienzos de la década de 1690-, que nada tiene que ver con la medicina, sino que es una colección de cartas que nacen de una denuncia pública a raíz de la escritura de un sermón con claros tintes heréticos, nacidos de la ignorancia de la materia y de la lengua portuguesa. ${ }^{19}$

${ }^{19}$ Se trata del manuscrito conservado en la Ets Haim con la signatura EH_48_e_28. Actualmente, el Prof. Den Boer y yo ya estamos preparando su edición. 
Resulta realmente interesante este ejemplar, ya que es una muestra más del carácter y el cariz que tenían las polémicas médicas que, en este contexto, surgían entre físicos y estudiantes. Dicho de otro modo, este breve texto estaría, pues, enmarcado en el conjunto de aquellas discusiones sobre teorías, diagnósticos y prácticas realizadas en algunos pacientes, cuyo objetivo, además de ganar renombre entre los círculos eruditos, era hacer nuevas aportaciones de carácter clínico y empírico a la ciencia.

El contenido de esta breve polémica parte precisamente del diagnóstico de una paciente que murió por diarreas biliosas, enfermedad y tratamiento al que Abraham Zacuto le dedicó un capítulo completo, concretamente la "Quaestio XLVI" del segundo libro De Medicorum Principium Historiae. Esto en sí no es un argumento para poder establecer vínculos con él, pero hay varios aspectos que sí permiten perfilarle como su posible autor. Es cierto que, teniendo en cuenta el texto precedente, resultaría un anacronismo, ya que Zacuto murió en 1642 y el resto de los textos que completan el ejemplar son de finales del XVII. Sin embargo, desde el punto de vista paleográfico, y estudiando la composición de los pliegues, ni la letra del amanuense coincide con el resto $-\mathrm{y}$ el texto que me ocupa aparece en unas cuartillas independientes, por lo que esto ya demuestra una autonomía con respecto al resto de los folios. Este hecho podría explicar, por un lado, que se tratase de un texto independiente y mal indexado y, por otro, no impide pensar en que fuese anterior al resto del compendio, más concretamente, de mediados de siglo.

Otro argumento a favor de la autoría de Zacuto (1642) sería el estilo de esta apología. Si antes he mencionado el carácter retórico y filosófico en sus obras, así como la cuestión ética y deontológica que desarrolla, basta con leer las primeras líneas para ver que esto también se cumple: "'Solo sé que no sé nada', dice el filósofo, sentencia digna de ser escrita en una lámina de oro y que en la memoria humana se mantiene viva, aunque otros son tan soberbios que 
estiman que con ellos nadie se puede comparar" (f. 52 r).$^{20}$ El texto está salpicado de referencias a fuentes clásicas, que, sin duda, permiten empezar a delinear a su posible autor. Pero más allá de esto, me interesa también fijarme en cuáles son los argumentos médicos que ofrece a -según dice en el texto- "in veni collegas iam iuxta portam exeuntes" y de dónde los extrae: son, en su mayoría, citas y razonamientos procedentes de los Aphorismos de Hipócrates y del De sanitate tuenda de Galeno, dos obras a las que remite de manera constante en el resto de sus trabajos y de las que, por cierto, no he encontrado rastro en los registros de los fondos bibliográficos públicos ni privados que se conservan de este contexto, pero que, contrariamente, seguro se conocían en los círculos académicos peninsulares (Sousa Dias, 2010, pp. 77-88). Esto lleva a otro punto, y es que, si se comparan las argumentaciones y razonamientos del manuscrito con lo que Zacuto postula en el capítulo correspondiente a las diarreas biliosas en su De Medicorum Principium Historiae, se puede ver que, efectivamente, son las mismas y que están en directa contraposición con las ideas y praxis que se recogen de los otros médicos. Esto se advierte concretamente en dos aspectos. El primero: los médicos, guiados por el hecho de que la paciente tenía fiebres, no supieron practicar las pertinentes sangrías, ya que ignoraron valorar los grados de calor del cuerpo, ni si se trataba de una fiebre ordinaria o una fiebre pútrida, lo que tuvo como consecuencia un grave desequilibrio de los humores corporales: en este caso, la sangre, fluido que rige al resto y, por consiguiente, a la bilis. En segundo lugar, y como resultado de lo anterior: esta descompensación entre humores no se debe remediar por medio de sangrías, porque, según responde a una intervención irreverente, que recoge de uno de los interlocutores, "Venae section debilitat

20 ''Scio me nihil scire', inquit Philos, sententia digna quod aurea lamina scribatur, it quod hominum memoria semper retineatur viva sunt namque, aliqui tam superbi quod existiment, neminem sibi ipsi posse comparari." La traducción es propia. Como otra muestra de socarronería: "Philosophi faciunt aliquas suppositiones de rebus, quas ipsi nesciunt, nec scire possunt; sunt namque hominibus occultae; si vero in hoc omnes, nemine discrepante non conveniant, non poterunt recte in suis disputationibus procedere, nec aliquid unquam concludere." 
vires; ergo si iterum mittatur sanguis magis debilitabuntur, et si sanguis iterum vena sesta ut unum imminuatur, et remanserit ut quatuor, vires etiam ex tua hypothesi imminuentur ut unum". Esta explicación también procede del De Medicorum Principium Historiae, por lo que, a partir de este breve análisis del manuscrito, considero que son varias las pruebas que demuestran una relación directa con Abraham Zacuto, y que evidencian su posible autoría.

Son varios los aspectos que, en conclusión, se pueden extraer de la revisión de estos ejemplares. Si bien es cierto que quedan fuera de esta relación nombres y obras de médicos, tan insignes como el afamado Rodrigo de Castro o Isaac Orobio de Castro, el interés era focalizar el estudio en los manuscritos, con el fin de, a partir de ellos, ver a qué uso estaban destinadas estas copias o notas, cuál fue su repercusión en su contexto y a qué fines estaban dedicados. En este sentido, se puede ver que, en la mayoría de los casos, eran epítomes realizados en gran parte por Benjamin Godínez, por orden de Isaac de Matatiah Aboab. De lo que no hay certeza es de quién realizó la selección de los fragmentos ni las traducciones, ya que, en ninguno de los casos, responden a reproducciones integrales de los originales. Sin embargo, todo apunta a que estos documentos estaban destinados a un uso personal, ya fuese como breves manuales de primeros auxilios, ya como pequeños cuadernos de notas, que servían de memorias de sus autores. Esto se sabe ya no sólo por la selección interesada de los fragmentos, sino por la lengua en la que están escritos: lo habitual es que este tipo de obras estuviesen redactadas en latín -tal y como sucede con los originales de los que surgen estas copias manuscritas-y que incluso los médicos se comunicasen en esta lengua, posiblemente porque se tratase de un contexto académico y profesional. Sin embargo, en todos los casos -salvo en el concerniente a la polémica que recojo- todos están redactados en portugués, idioma que, en la mayoría de las ocasiones, estaba relegado a un uso familiar y, en cierto modo, coloquial.

Sea como fuere, de lo que sí se tiene constancia era de un claro conocimiento de cuáles eran las praxis más aceptadas, ya no sólo entre los profesionales de la comunidad, sino a nivel general. Muestra de ello es que tanto el Catálogo de diferentes remedios como 
el Libelo aureo da dificultade de ourinar recogen las teorías de doctores tan eminentes como François Collot -uno de los más respetados por los especializados en litotomías y en el sistema renal- o Rodrigo de Castro -el padre de la ginecología moderna-, de los cuales, además, ofrecen garantías, ya que presentan evidencias hermenéuticas, deducidas de intervenciones a pacientes, de los que dan toda información. En este sentido, se aleja un tanto la obra de Zacuto, no por su cuestión médica, que fue a menudo alabada, sino por las interferencias de carácter meta-medicinal, que atienden a cuestiones deontológicas, morales y, en algunos casos, religiosas, a lo que hay que sumar la inserción de citas literarias clásicas y valoraciones filosóficas. Estos aspectos le valieron para ser el centro de las críticas de varios contemporáneos, ya que excedía las barreras de lo científico. No debió ser de la misma opinión Isaac de Matatiah, quien, en los paratextos que acompañan las notas médicas, ofrece una buena cantidad de consejos, que estriban entre los principios éticos hipocráticos y la moral judaica.

Las características que marcan, en buena medida, el manuscrito relativo a la obra de Abraham Zacuto sirve de pistas para acotar otro ejemplar del que, hasta ahora, no ha habido noticia, salvo por sus primeras hojas, dedicadas a recoger una apología en detracción de la herejía. Centrado en la práctica de las sangrías en casos con fiebres ordinarias o pútridas y diarreas biliares, el autor de la breve apología médica desarrolla una crítica satírica y sarcástica contra aquellos que las realizan de manera irregular y, evidentemente, contraria a su teoría. El interés que presenta es que, además de esta controversia clínica -escrita en latín, por lo que se entiende que debió concebirse en un plano académico o profesional-, su autor apoya sus argumentaciones y su ironía en fuentes clásicas, tanto es así que, si en el plano científico se presenta como un claro defensor de la tradición hipocrática, en el aspecto de la detracción se surte de tópicos filosóficos para hacer escarnio, de marcado corte humanístico, del mal proceder de algunos de los médicos de su entorno. 
АвОАВ, D. (1685). Catálogo de diferentes remedios para diversas sortes de achagues achados por experiencia, haverem sido boms. Ms. de la Ets Haim (eH 48 e 14).

Arrizabalaga, J. (2007). The World of Iberian Converso Practitioners, from Lluís Alcanyís to Isaac Cardoso. (V. Navarro Brotóns y W. Eamon, Eds.). Más allá de la Leyenda Negra: España y la Revolucion Cientifica/Beyond the Black Legend: Spain and the Scientific Revolution (pp. 307-322). Valencia: Universitat de Valéncia.

Arrizabalaga, J. (2009). Medical Ideals in the Sephardic Diaspora: Rodrigo de Castro's Portrait of the Perfect Physician in early Seventeenth-Century Hamburg. Medical History, 53(29), 107-124. $<$ https://www.ncbi.nlm.nih.gov/pmc/articles/PMC2836222/>.

Baudry, H. (2020). Medical Publishing in Portugal in the First Half of the Seventeenth Century: A Good Business? En A. S. Wilkison y A. Ulla Lorenzo (Eds.), A Maturing Market: The Iberian Book World in the First Half of the Seventeenth Century (pp. 225240). Leiden: Brill.

Ben Israel, M. (1633). Conciliator sive de convenientia locorum S. Scripturae quae pugnare inter se videntur. Frankfurt: Auctoris typis \& impensis.

Berger, S. (1996). Classical Oratory and the Sephardim of Amsterdam: Rabbi Aguilar's Tratado de la retórica. Hilversum: Verloren.

Bergmann, A. (2006). Ets Haim: Tradition and Innovation in Jewish Education. Columbia: Columbia University Graduate School of Arts \& Sciences.

Bizouard, J. (1863). Des rapports de l'homme avec le démon. Essai historique etphilosophique. París: Gaume frères et J. Duprey.

Brown, K. (2000). Spanish, Portuguese, and Neo-Latin Poetry Written and/or Published by Seventeenth, Eighteenth, and Nineteenth-Century Sephardim from Hamburg and Frankfurt. Sefarad, 60(2), 227-253.

Cohen, D. E. (1930). De Amsterdamsche Joodsche chirurgijns. Nederlands Tijdschrift voor Geneeskunde, 74(18), 2234-2257. 
Da Silva Rosa, J. s. (1925). Geshiedenis der Portugeesche Joden te Amsterdam. Ámsterdam: Menno Hertzberger.

De Barrios, M. (1683). Triumpho del gobierno popular. Ámsterdam: David de Castro Tartás.

Den Boer, H. (1995). La literatura sefardí de Ámsterdam. Alcalá de Henares: Instituto Internacional de Estudios Sefardíes y Andalusíes.

Franco Mendes, D. (1976). Memorias do estabelecimento e progresso dos judeos portuguezes e espanhois nesta famosa cidade de Amsterdam. (L. Fuks y R. G. Fuks-Mansfeld, eds.). Ámsterdam: Assen.

Friedenwlad, H. (1939). Abraham Zacutus. Bulletin of the History of Medicine, 7(5), 458-485.

Fuks, L. \& Fuks-Mansfeld, R. G. (1975). Hebrew and Judaic Manuscripts in Amsterdam Puvlic Collections. Leiden: Brill.

HARRIS, C. (2008). The way Jews lived: five hundred years of printed words and images. North Carolina/London: McFarland.

Jarcho, S. (1989). The style of Zacutus Lusitanus and its Origins. The Journal of the History of Medicine and Allied Sciences, 44, 291295.

Kayserling, M. (1890). Biblioteca Española-Portuguesa-Judaica. Dictionnaire bibliographique des auteurs juifs, de leur ouvrages espagnoles et portugais et des oeuvres sur et contre les juifs et le judaïsme avec un apercu sur la littérature des juifs espagnols et une collection des proverbes espagnols. Strasbourg: B. de Graaf.

LeÃo Benavente, S. (1690). Libelo aureo da difficultade de ourina. Ms. de la Ets Haim (EH 48 D 32).

Lemos, M. (1909). Zacuto Lusitano: a sua vida e a sua obra. Porto: E. Tavares Martins.

Librach, I. M. (1960). Medicine and Manasseh Ben Israel. Medical History, 4(3), 256-58.

Löwenstein, L. (1904). Die Familie Aboab. Monatsschrift für Geshichte und Wissenschaft des Judentums, 48, 661-701.

MacLean, I. (2009). Learning and the Market Place: Essays in the History of the Early Modern Book. Leiden/Boston: Brill.

McGana, M. (1998). The Story of Joseph in Spanish Golden Age Drama. London: Associated Universty Press. 
Moreno de Carvalho, (1996). I am a wandering Jew: The judaic context of the intellectual life of the Physician Zacuto Lusitano (en hebreo). Periodical of the World Association for Judaic Studies, 36, pp. 147-159.

Moreno de Carvalho, F. (1999). Zacuto Lusitano e um Tratado de Medicina Dirigido ao Brasil. En N. Falbel, A. Milgram, A. Dines \& E. Lipiner (Eds.), Em Nome da Fé. Estudos in Memoriam de Elias Lipiner (pp. 57-74). São Paulo: Editora Perspectiva.

Oliel-Grausz, É. (2017a). A Tale of Caribbean Deviance: David Aboab and Community Conflicts in Curaçao. En The Religious Cultures of Dutch Jewry (pp. 157-182). Leiden: Brill.

Oliel-Grausz, É. (2017b). David Aboab, ou l'itinéraire frustré d'un juif converti au XVIII ${ }^{\mathrm{e}}$ siècle entre lacunes et certitudes. Revue de l'Histoire des Religions, 1, 89-118. <https://doi.org/10.4000/ rhr.8656>.

PAncorbo, F. J. (2021). Los Documentos para todo estado e ydade de Ishack de Matatia Aboab: De las lecturas moralizantes a la judaización de la Paraenesis ad Demonicum. (F. J. Pancorbo y H. den Boer, Eds.). El canon del converso: nuevas aportaciones literarias (pp. 33-49). Santa Bárbara: Humanista.

RÉvaH, I. s. (1961). Pour l'histoire des nouveaux-chrétiens portugais. La rélation généalogique d'Isaac de Matatiah Aboab. Boletim de bibliografía luso-brasileira, 2, 276-310.

Roth, C. (1979). Los judios secretos: Historia de los marranos. (Trad. de J. Novella). Madrid: Altalena.

SCHLeIner, W. (1995). Medical Ethics in the Renaissance. Washington: Georgetwon University Press.

Sousa Dias, J. P. (2010). Até que as Luzes os separem: Hipócrates e Galeno na literatura médico-farmacêutica portuguesa dos séculos XVII e XVIII. (A. Vanda e I. de Ornellas e Castro, Eds.). Revisitar os Saberes: Referências Clássicas na Cultura Portuguesa do Renascimento à Época Moderna (pp. 77-88). Lisboa: Centro de Estudos Clássicos-Universidade de Lisboa.

SzAnCER, H. (1967). Introduction à la 'Pharmacopea elegantissima' d'Abraham Zacutus Lusitanus. Revue d'histoire de 
la pharmacie, 194, 509-514. <https://www.persee.fr/doc/ pharm_0035-2349_1967_num_55_194_7664>.

VAN Esso BZN, I. (1940). Net aandeel der Jooden in de natuurwetenschappen in de Nederlanden. (Brugman y Frank, Eds.). Geschiedenis der Joden in Nederland, I, pp. 643-679.

Zacuto, A. (1642). Opera omnia. Lyon: Joannis-Antonii Huguetan.

Zacuto, A. (1690). Tratado sobre Medecina que fez o doutor Zacuto para seu filho levar consigo quando se foy para o Brazil. Disposto e copeado por bordem de Ishak de Matatia Aboab. Anno 5450. Escrito por Benjamin Godines. Ms. de la Ets Haim (EH 48 e 45). 